\title{
Visual Confidences and Direct Perceptual Justification ${ }^{1}$
}

Abstract: What kind of content must visual states have if they are to offer direct (non-inferential) justification for our external world beliefs? How must they present that content if the degree of justification they provide is to reflect the nuance of our changing visual experiences? This paper offers an argument for the view that visual states comprise not only a content, but a confidence relation to that content. That confidence relation explains how visual states can offer direct perceptual justification of differing degrees for external world beliefs. These confidence relations allow that visual states justify beliefs in a way that is sensitive to subtle differences in the character of our visual experiences, whilst still allowing that visual states give us non-inferential access to the external world in virtue of their content.

Visual states justify beliefs. My belief that there is a turtle on the beach is justified by a visual experience that represents a turtle. But not all experiences that represent a turtle are created equal: whilst some offer excellent justification for belief, others offer only weak justification, as when the turtle is seen at a distance. Although far and near experiences may both represent a turtle on the beach, the particular appearance of the turtle in each instance varies. And that variation has important ramifications for the capacity of the experience to justify belief. An adequate account of perceptual justification must be capable of accommodating and explaining that variation. It must link the way the world appears, to the justification our perceptual beliefs enjoy.

How are we to explain this variation in perceptual justification? What properties must our experience have if it is to account for these fluctuations in epistemic power, even across experiences that share epistemically relevant contents? I propose that we should understand the relevant difference in such cases as a change in the certainty or confidence with which the visual state presents its content. Doing so allows that direct visual justification for beliefs about the external world can come in degrees that reflect subtle but epistemically relevant shifts in phenomenology.

In section one I use two cases to focus our attention on the question in hand: what feature of visual states explains how they can offer justification of different degrees to belief? In section two I suggest that these cases reveal a tension between four intuitive principles in perceptual epistemology. In section three I survey possible avenues of responses to

${ }^{1}$ Acknowledgements 
that tension. I suggest that the source of the tension is the assumption that visual states are not graded. In section four I describe an alternative view, according to which visual states too come in degrees. Section five offers an explanation of how graded visual confidences can account for the degree of justification visual perceptual states confer on beliefs, including graded belief states. Section six uses visual confidences to explain how visual experience can offer us direct justification for more complex beliefs, including comparative and disjunctive beliefs.

In what follows I shall focus on visual experiences. The argument may generalize to perceptual experience and perceptual justification more broadly. Since I think it important we leave open the possibility that there may be important discontinuities between visual justification and perceptual justification via other sensory modalities, I will talk in terms specific to vision.

\section{Elmer and the Fog}

The phenomenal character of a visual experience can change incrementally. How things look to you develops gradually as you move, as the scene you are looking at evolves, or as environmental conditions change. Those changes in phenomenal appearance have epistemic upshots: your epistemic position also changes very gradually, in line with them.

It is natural to think the epistemic power of an experience depends at least in part on its content: ${ }^{2}$ whether or not a given experience can justify a belief depends on whether the experience has the appropriate content. This raises the following question: what form must the content of experience take, or how must it be presented, such that it can reflect these subtle changes in appearance, and their impact on the justification we have for belief?

Consider the following cases:

Fog: Elmer is sitting inside a house while the fog clears outside. At some point he will get up and look out of the window at a tree which stands outside. He could have a range of visual experiences, depending on the point at which he looks out the window. If he goes to the window soon the tree will be wholly obscured by fog. If he waits an hour, he will have a perfectly clear

2 This paper engages with a tradition that assumes experiences have representational, truth-evaluable contents. For recent denials of this claim see Travis 2004, Brewer 2006. 
experience of the tree. In between those times there is a continuum of available experiences, from very-foggy to no-fog-at-all.

Turn: Rey is in the process of turning her head. As she turns her head, a medium-sized grey box will come into her view. First she sees the box only out of the corner of her eye. As she continues to turn, her visual experience of the box becomes clearer. By the time she has completed the movement she will be looking straight at the box.

What do we want to say in these cases? If Elmer looks out of the window in an hour's time, once the fog has cleared, his visual experience is well placed to offer him direct justification for the belief that there is a tree outside the window, just as Rey's experience once she has completed the turn may directly justify the belief that there is a large grey box some particular distance in front of her. Intuitively, Elmer and Rey's epistemic positions change gradually. If Elmer goes to the window now, he will enjoy weak justification for the belief that there is a tree outside. And if he goes to the window in an hour, he will enjoy excellent justification for the same belief. But the change between those points is incremental: any small change in the amount of fog should not make a big difference to the degree of justification he has for a tree belief. Rather, the epistemic change between points is gradual, just as the change in the appearance of the tree is gradual.

These cases focus our attention on the visual states responsible for the justification of the relevant beliefs. What properties of those states could explain this subtle variation in degree of justification? How do those states change as the fog clears? And at what points can Elmer or Rey receive direct justification for their beliefs?

We could ask the same question not in terms of degree of justification but in terms of degree of belief. Rey should be more confident in her beliefs about the box's location when she is looking straight at it, than when she sees it from the corner of her eye. As she turns her head, the confidence her experience is capable of justifying gradually increases. What feature of the visual experiences Rey has explains the change in the confidence she is entitled to have in the location of the object? And which of those confidences is directly justified by her experience?

Note that at some level the contents of Rey and Elmer's experiences may remain the same, even as their phenomenal character changes in a way that is epistemically relevant. Suppose some of Elmer's experiences 
include tree content. ${ }^{3}$ Even when he sees the tree through some limited degree of fog, his experience may still include tree content. But it is consistent with this that as the fog becomes thicker, and as the character of Elmer's experience changes to reflect that, the degree of justification his experience provides for a tree belief begins to wane. What is it about the visual state then that explains that variation in justification, if it isn't the simple presence or absence of tree content?

The kind of variation in perceptual appearance we are interested in is not confined to fog. Our visual experiences are richly nuanced. How an object looks to us depends on its distance from us, whether it is seen fleetingly or at an angle, the lighting conditions, and facts about our own visual system, such as whether we are wearing necessary corrective glasses or not. And yet a wide range of differently nuanced experiences may represent the same object. In this way, those factors may not be sufficient to change the epistemically relevant content of the experience - I may still have a visual experience that represents a glass a particular distance from me whether I see it clear eyed at the start of the evening, under the influence at the end of the evening, or blearily first thing in the morning. Despite their shared content ( $x$ is a glass), and the shared content of the beliefs they justify, those experiences provide different degrees of justification. That difference is tied to their phenomenology. What, then, are the epistemically relevant properties of visual states that explain that gradual change in degree of justification?

\section{Principles}

I suggest that cases like those of Elmer and Rey reveal a tension between the following four principles.

(1) Direct Visual Justification (External World): Some external world beliefs are directly justified by visual experience.

(2) Content: a visual experience directly justifies belief in virtue of its contents.

(3) Degree: Direct visual justification comes in degrees.

(4) Binary: Visual states are not degreed.

\footnotetext{
${ }^{3}$ Note that these cases do not rely on the assumption that visual states include "high-level" content at the level of ordinary objects like trees or glasses. See below in section three for an argument that they can arise even for low-level content.
} 
I shall argue that we should abandon (4), accepting instead that visual states present their contents with varying degrees of confidence. I turn now to a discussion of each of these principles.

What properties must visual states have if they are to offer direct yet degreed justification? This question starts from the basic and intuitive claim that visual experience gives us direct justification for beliefs about the external world.

(1) Direct Visual Justification (External World): Some external world beliefs are directly justified by visual experience.

Direct justification is non-inferential. It stands in contrast thereby with indirect justification, when a visual experience justifies a belief in virtue of the good standing of additional inferential steps that intervene between the experience and the belief.

Suppose you see a light on in the kitchen window as you arrive home. That could justify a belief that your partner is already home. But that justification is indirect. You infer from the visual experience of the light that your partner is home. When you have a visual experience of your partner at home, then your belief is a candidate for direct justification. ${ }^{4}$

DVJ (ExW) underwrites foundationalist accounts of justification which claim that perceptual experience can offer a basic kind of justification for belief, one that allows us to begin the process of gathering the information which may then features in the inferences or interpretive priors that ground the justification of further beliefs. These accounts claim not just that perceptual experience justifies belief, but that it can provide immediate justification for belief, that is, justification that does not rely on the subject possessing justification for any other beliefs (Alston 1989, Audi 1993, Pryor 2005, Huemer 2001, Feldman 2003). ${ }^{5}$

The basic distinction between direct and indirect perceptual justification is this: some beliefs are justified because of a relation that holds directly between them and a visual experience. Other beliefs are

\footnotetext{
4 Siegel 2006 draws a related distinction between what is presented perceptually, and what we go on to believe.

${ }^{5}$ Direct justification by experience need not be immediate in this way. You could think that visual experience provides direct justification for certain beliefs only once other ancillary conditions are met, for instance, that the relevant perceptual process be sufficiently reliable (see Goldman 2006 for an example of such an account). It is possible that some beliefs are directly justified by experience even if none are immediately justified
} 
justified in virtue of the good standing of further inferences that mediate between the experience and the beliefs that can be based upon it.

$\mathrm{DVJ}(\mathrm{ExW})$ is a controversial view in perceptual epistemology, but it is an important one. It captures a simple and intuitive picture of how we come to learn about the external world: namely that we do so directly, via our sensory experience. This simplicity offers it a certain power: as a consequence the view can avoid certain skeptical arguments. ${ }^{6}$ According to dogmatist or phenomenal conservative views that entail DVJ (ExW), at least some of our beliefs enjoy prima facie justification on the basis of experience in a way that leaves them immune to the introduction of skeptical doubts about the good standing of our knowledge about the

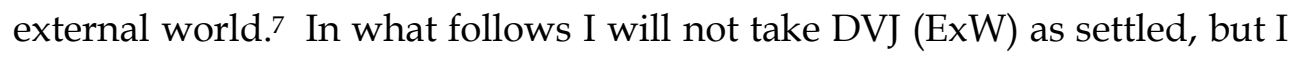
will assume that it represents an important and appealing position, one that is worth defending, if possible.

How do we circumscribe the set of beliefs which can be directly justified by visual experience? ${ }^{8}$ In virtue of what does a visual experience directly justify a belief? It is natural to answer both those question in terms of relations between the contents of the two states. The set of beliefs capable of immediate justification by visual experience is the set of beliefs whose content is appropriately related to a visual experience. ${ }^{9}$

We can leave open at this stage what it takes for the content of a belief and an experience to be appropriately related. At its strongest once could think of the relation in terms of the uptake of visual contents by belief: there is an analogue of visual contents for belief, and a visual experience simply justifies the set of beliefs with the belief-analogue of its contents. ${ }^{10}$ More loosely, we could think of belief as endorsing the content of experience, 11 or of belief that is a "proper response" to an experience. ${ }^{12}$ Common to these approaches is the insight that we explain the capacity of

${ }^{6}$ See Pace (2010) or McGrath (forthcoming) for this point.

7 As for instance in Pryor (2000), Huemer (2001).

${ }^{8}$ Rescorla (2014) terms this question the "demarcation problem". He attributes the observation that dogmatist approaches to justification encounter it to Wright (2007).

${ }^{9}$ Note, though, that visual experience is not a prerequisite for direct justification, nor is the class of directly justified beliefs restricted to contentful beliefs.

Introspective beliefs about one's mental states or bodily sensations may be immediately justified by those states, even when those states fail to have any content, as is arguably the case with pain, for instance (Pryor 2005)

10 This "uptake" picture is closely related to the content constraint critiqued in Silins (2011), according to which "[i]f your visual experience $e$ gives you immediate justification to believe some external world proposition that $p$, then it is a phenomena content of $e$ that $p^{\prime \prime}$ (Silins 2011:335).

11 See for instance Chalmers (2010), or Siegel (2013) for this usage.

12 Richard Feldman (2003) draws on a notion of content closeness to develop an account of belief which is a "proper response" to experience. 
an experience to directly justify some beliefs but not others in terms of its content.

(2) Content: a visual experience directly justifies belief in virtue of its contents.

The way in which direct visual justification relies on a relation between the contents of experience and belief limits the kinds of beliefs that can be directly justified by perceptual experience to those whose content is capable of standing in that appropriate relation to visual content. What sorts of contents experiences have therefore constrains which beliefs can be directly justified. ${ }^{13}$ Unless you think visual experience can include contents like "a successful and eccentric lothario," it is unlikely that your belief that there's a successful, eccentric lothario in front of you can be directly justified by a visual experience. ${ }^{14}$ You might instead gain visual justification for such a belief on the basis of inferences drawn from more basic beliefs, for instance about the individual's appearance, beliefs which are directly justified by the visual experience.

What properties and objects can feature in visual experiences is the subject of intense debate. Some will think that those contents are restricted to color, size and location properties. Others will be happy to include richer contents, including causation, agency or certain natural kind properties. ${ }^{15}$ If visual experiences include richer contents then they are capable of directly justifying a wider range of external world beliefs.

Note that a single visual experience may include a range of contents, not all which may be relevant to the justification it provides for a given belief. Suppose I have a visual experience of a red ball against a backdrop of mountains and sky. I form the belief "that's a red ball". Relative to this belief, the visual contents of the red ball is the operationally relevant content, since it is those contents which render the belief a good candidate for direct justification by the visual experience. The backdrop of mountains and sky, by contrast, are not.

So far so good: some external world beliefs are capable of being directly justified by visual experience, namely, those beliefs whose content is appropriately related to the content of a visual experience. But in addition to which beliefs we have justification for, our perceptual

\footnotetext{
13 Siegel and Silins (2015) refer to this direction of constraint as the "reverse content constraint".

14 See also Richard Feldman (2003) for this point, made using the example of an eighty-four year old table.

15 See for instance Siegel (2006), Bayne (2009), Hawley and MacPherson (2011), Brogaard (2013) for recent discussion of this question.
} 
justification can vary along another axis: the degree of justification we have for a given belief. Sometimes, when things look one way to you but another to me, our experience may provide us with different degrees of justification for the same belief. You saw the turtle on the beach from right up close, so your belief that there's a turtle on the beach is very strongly justified. I saw the turtle at a distance. I have justification for the same belief, but I have less or weaker justification. ${ }^{16}$

(3) Degree: Visual justification comes in degrees.

Degree is supported by the intuition that the visual justification we receive should be responsive to variations in how things look to us. If something looks one way to you, but another way to me, then you may have justification I lack, and vice versa. Those variations in our phenomenal experience explain why your belief is more strongly justified than mine. Since those phenomenal differences are subtle and degreed, justification too must be degreed if it is to capture them.

This intuition in effect sets a desideratum on accounts of perceptual justification: they should allow that the justification visual experiences provide for belief reflects the changing nuance of our perceptual phenomenology. It's natural to think both that phenomenal character constrains perceptual justification by limiting which beliefs you have justification for, and that it also plays a role in determining the degree of justification those beliefs enjoy.

Desideratum: the justification a visual experience $E$ offers for belief reflects the phenomenal character of $E .17$

16 See Pautz 2011, and 2015 and Schellenberg 2015 for a more recent instance of the claim that perceptual justification comes in different degrees. Alvin Goldman makes a similar point in discussing process reliabilism: "Suppose Jones believes he has just seen a mountain-goat. Our assessment of the belief's justifiedness is determined by whether he caught a brief glimpse of the creature at a great distance, or whether he had a good look at the thing only 30 yards away. His belief in the latter sort of case is (ceteris paribus) more justified that in the former sort of case..." (Goldman 1979:10).

17 One way of respecting that desideratum is via a principle connecting phenomenal character and phenomenal content. If phenomenal content grounds phenomenal character, for instance, such that any change in phenomenal character corresponds to a change in phenomenal contents, then as a result of Content, any change in phenomenal character corresponds to an epistemically relevant difference in an experience. Many representationalists endorse the claim that content grounds character in this way (Dretske (1995), Hill (2014), Schellenberg (2014), and Tye (1995)). That principle on its own, however, leaves unanswered the question of what the relevant change in contents is in these cases. 
Experiential contents are stretched between different theoretical demands imposed by the principles above. On the one hand, DVJ (ExW), coupled with Content requires that the operationally relevant contents of experience be at a level that feature in our ordinary, external world beliefs. These beliefs are fairly thick grained: they feature objects and properties. On the other hand, in order to respect Degree, those operationally-relevant contents need to be sufficiently fine-grained that they are capable of accommodating the variation in visual justification introduced by subtle variations in the character of our experience. What properties must visual states have, if they are to directly justify external world beliefs, whilst allowing that that justification comes in differing degrees?

It is often assumed that visual states are not degreed. There are two ways in which visual states could be degreed. On the one hand, the contents themselves could be degreed: visual content could be probabilistic, for instance. Alternatively, or in addition to this, visual states could include a degreed relation to their content. If neither of those possibilities hold, then visual states involve a binary relation to a binary content. ${ }^{18}$ On this picture, visual states are all-or-nothing. An experience includes an item among its contents, or it fails to do so. In this way, this assumption about visual states comes apart from what is increasingly becoming the standard model of belief, which allows that at least some belief states are graded. On these models of belief you do not simply believe or fail to believe a proposition. Instead you bear a graded confidence relation to a belief contents. By contrast, binary visual states do not come in degrees in either of the ways described above. ${ }^{19}$

(4) Binary: Visual states are not degreed.

These four principles are in tension with one another. To see this tension, return to the case of Elmer. On the one hand, we want to say that some of Elmer's visual states can directly justify his tree belief. On the other hand we do not want to say those visual states offer the same degree

\footnotetext{
18 It is sometimes said that visual representations can be indeterminate (Pautz 2011, Siegel 2006b , Block 2010). But as I argue below in section 3.5, unless that determinacy is degreed then it will not be able to provide the resources we need. I regard degreed indeterminacy as a close relation of the solution I myself propose. 19 Recent work by John Morrison bucks this trend. Morrison argues in favor of a view he calls "perceptual confidence", that "our perceptual experiences assign degrees of confidence" (forthcoming: 2). Sarah Moss (ms. section 4.3) also offers arguments in favor of the view that some perceptual experiences have what she describes as probabilistic contents.
} 
of justification as one another for that belief. Assuming his visual states must be binary makes it hard for us to reconcile those desiderata.

Suppose that visual experiences can include tree content, such that beliefs about trees can be directly justified by visual experience. At least some of Elmer's experiences include tree content. Those experiences with tree content can directly justify tree beliefs in virtue of that content, in line with Content, and DVJ (ExW). The experience at the no-fog end of the spectrum couldn't be any clearer, so if any experiences have tree content, this is a good candidate. Let's call this experience $E_{t 1}$. The experience at the other end of the continuum has no tree content. Let's call that experience $E_{t \infty}$.

How far does the direct justification of tree beliefs reach down that continuum of experiences? Take an experience very shortly after $E_{t 1}, E_{t 2}$. This is distinguished from $E_{t 1}$ by a very tiny amount of fog. This quantity of fog is so slight it barely impacts on the phenomenal character of the experience. If $E_{t 1}$ includes tree content, then it is implausible that $E_{t 2}$ does not include it. If $E_{t 1}$ can directly justify a tree belief, then so can $E_{t 2}$.

At some point in the chain of experiences, as the fog becomes thicker, the degree of justification Elmer's experience provides for a tree belief begins to wane. If Elmer were to phone a suspicious friend to report his belief that there's a tree outside the window, the suspicious friend might interrogate the conditions under which he saw it, to try and establish just how well justified Elmer's belief is. And on hearing that he saw it through fog, he could reasonably judge that Elmer's belief about the tree was not as well justified as if he'd seen it on a clear day.

Direct justification by visual experience is grounded in visual contents. The kind of nuance in phenomenal character introduced by fog, or distance, or movement, or mildly defective occular hardware, may not be sufficient to change the operationally relevant content of the experience. I may still have a visual experience that represents a tree whether I see it through a little fog or on a sunny day. And according to Binary, those visual states do not come in degrees. So what, then, is the change in the visual state that means that the same belief is justified to a lesser degree, depending on the time at which Elmer walks to the window?

This problem is only intensified when we pose it in terms of the justification of credal rather than binary belief states. The question becomes, what must visual states be like if they are to be capable of directly justifying different degrees of credence in a particular proposition? What explains why in some cases an experience entitles the subject to be highly confident in a given belief, and in others to have only a low confidence? Is 
there a way of explaining that variation consistent with the claim that that entitlement is non-inferential?

At the heart of all of these questions is the need for an account of how belief and experience interface with one another. It becomes increasingly hard to offer such an account, and hence to make sense of direct justification in virtue of experiential contents once we bring credences into the picture. Yet degrees of belief are an increasingly central way of modeling belief-states in both philosophy of mind and epistemology. It is natural to understand the contents of belief and perceptual states as structurally analogous to one another. This is motivated in part by the way in which the two seem capable of interacting with one another. But if belief comes in degrees, whilst perceptual states stand in a binary relation to their contents, how do they interface with one another? What facts about an experience fix the confidence a subject is entitled to have in beliefs in its contents?

Perhaps we can sidestep this question by allowing that a believer is entitled to uptake the content of all visual experiences with credence one. A visual experience of a tree supports a belief with credence one that there is a tree at the relevant location. An experience of a tree shrouded in some degree of fog supports credence one in the belief that there is a tree in that location, shrouded in light fog. This approach supposes that we trust absolutely the determinate deliverances of our experience, and that those deliverances hence support the strongest credence.

But there are good reasons to think that we don't give credence one to the deliverances of our senses. Beliefs to which we give credence one are immune to revision. As rational actors we should be willing to accept any bet against them, however high the stake and however small the gain. That does not seem like a plausible description of our relationship with our sensory evidence. In fact, some have argued that it is implausible to think we give any empirical propositions credence one. ${ }^{20}$ Direct justification is still defeasible justification. We frequently do revise our estimation of what we have seen, and just as often express our relationship with the evidence of our senses in uncertain or probabilistic terms.

If we assign these propositions a confidence of less than one on the basis of a visual experience, what fixes the appropriate credence? Consider Richard Jeffrey's example of an ambiguously colored cloth: "The agent inspects a piece of cloth by candlelight, and gets the impression that it is green, although he concedes that it might be blue or even (but very improbably) violet" (Jeffrey 1965:165). Jeffrey is concerned with the

${ }^{20}$ For discussion see Christensen 2004, or Frankish 2009. I set to one side the Williamsonian tradition which equates evidence and knowledge (Williamson 2000). 
difficulties this raises for Bayesian conditionalization, which assumes that the evidence on which we update is accorded a probability of one. Jeffrey conditionalization is a response to this. It allows that the evidence on which a subject updates need not have a probability of one. But this raises a prior question which has so far gone unanswered: what determines the probability that visual evidence receives? How does that evidence take a graded form, if the experiential state itself is binary? There is a mismatch between the form of the visual state and that of the belief-state that responds to it.

These questions focus our attention on the nature of visual states and their content. Is there a way for visual states to offer justification for external world beliefs that is both direct, and degreed?

\section{Responses}

I turn now to surveying five possible responses. The first two allow visual experience to offer degreed justification by abandoning or restricting $D V J$ $(E x W)$, the claim that visual experience directly justifies external world beliefs. The next two preserve DVJ (ExW) by offering an alternative set of operationally relevant contents. The intention of this section is not to offer a definitive dismissal of these possibilities, so much as to survey the available territory, and the costs associated with taking one or other route into it. The fifth option moves us towards the solution I endorse by querying the assumption that visual contents must be determinate. In the next section I go on to develop one particular version of this response, which gives up the claim that visual states are binary, allowing instead that they included graded confidence levels. This lets us capture the epistemic impact of subtle changes in our phenomenal experiences without demanding a retreat from the claim that visual experience can directly justify external world beliefs.

\section{i. Appearance properties}

One natural response to these sorts of cases is to think that we have misdescribed the sort of beliefs that can be directly justified by experience, in virtue of misidentifying the set of operationally relevant perceptual contents. According to this approach, the sort of properties that feature in visual experience and in virtue of which experience directly justifies belief are appearance properties. The beliefs they license are beliefs about how the world appears to us. From those beliefs about appearances we can then justifiably draw inferences about the external world. An object that looks like a cat is likely to be a cat. Differences in the strength of the inference 
from the appearance property to the belief in question are responsible for the resultant degree of justification our external world beliefs enjoy. An inference that an object that looks like a cat is a cat is stronger than an inference that an object that looks like a cat is a Siamese cat, for instance.

On this account, Elmer enjoys direct justification on the basis of his experience for a set of beliefs about how the object in his visual field appears to him. His belief "that's a tree" is justified in virtue of the good standing of the inferences he draws that an object with those appearanceproperties is likely to be a tree. As the fog gathers that inference from his appearance belief to his external world belief becomes increasingly tenuous and so the degree of justification he enjoys for it decreases.

A similar retreat is open to us if we think that belief is degreed. We can treat the beliefs which are in receipt of justification as introspective beliefs about experience, which then receive credence one. Though I may be unsure of the color of the cloth, I can be sure that the cloth looks this way to me. Coupled with relevant background information, that belief about how things seem to me can in turn justify an appropriate credence in the belief that the cloth is blue or purple. I can then update the rest of my beliefs accordingly.

This style of response constitutes a retreat from DVJ (ExW) to a weaker form of the claim that visual experience offers direct justification for belief. That weaker claim is

DVJ (Internal World): Only beliefs about one's own experience are directly justified by visual experience,

We observed above that DVJ $(E x W)$ is attractive in part because of the role that it plays in supporting accounts according to which visual experience offers a foundational kind of grounding for belief. We can distinguish between two kinds of foundationalism, "classical foundationalism" and "modest foundationalism". ${ }^{21}$ Classical foundationalism allows that only beliefs about the character of one's own experiences are foundationally justified. Modest foundationalism allows that beliefs about the external world can enjoy foundational justification. In retreating from $D V J(E x W)$ to DVJ (IntW) this response to cases like those of Rey and Elmer in effect throws in the towel on a certain epistemological project, one which, in line with modest foundationalism, has ambitions to establish that our perceptual experiences put us in contact with the external world directly. ${ }^{22}$

${ }^{21}$ See Pace (2010) and McGrath (forthcoming) for this distinction.

22 Though see McGrath (forthcoming) for a view on which visual experience directly justifies "looks propositions", but according to which those propositions are external world propositions 
Those ambitions in turn offer these accounts the resources to oppose skeptical arguments that intervene on the inferential step between appearance and external world beliefs. Whilst acknowledging that retreating to appearance properties offers one potential avenue of response to these cases, it is worth continuing to look for responses to these cases that let us preserve DVJ (ExW).

\section{ii. Very few beliefs are directly justified by visual experience}

Another possible response to the tension described above claims that only the very clearest experiences have tree content of the kind that can directly justify tree beliefs. After that, Elmer's beliefs are justified indirectly. For instance, a belief that there is a tree outside could be justified by an experience with the demonstrative content that the scene outside the window looks like this, and an inference reliant on additional information about what trees look like when seen through slight fog. In Elmer's case, this response claims that only the clearest experience has tree content of the kind that can be directly taken up in belief. There is a shift in the operationally relevant experiential contents as soon as any amount of fog accrues.

In effect, this response admits two distinct kinds of justification relation - one that is direct, and one that is degreed. Since the kind of justification that comes in degrees is not direct, the visual states that provide direct justification need not also be capable of reflecting shifts in degree of justification. As a consequence, some external world beliefs are directly justified by visual experience, but only very few. Couched in terms of credences, this approach translates to the claim that the clearest experiences directly justify credence one. Other lower-level credences are arrived at inferentially.

The kind of inferential story offered in this response plausibly captures what is happening towards the foggier, $E_{\text {to }}$ end of the spectrum. But anyone with an investment in DVJ (ExW) should hesitate before restricting the reach of direct justification to only the clearest cases, or the highest credence. The first reason is that doing so would amount to limiting it to so tiny a set of cases that it circumscribes the epistemic role it can play. We have optimally clear experiences very rarely, if ever. We almost always see objects under conditions that are suboptimal, however marginally, on some axis or other: distance, lighting, weather, occluders or our own eye-sight. If all these shifts in phenomenology are incompatible with direct justification, then direct justification is largely redundant in explaining how our visual beliefs are formed and justified. Equally, as we saw above there is good reason to think we rarely, if ever, have credence 
one in a visual belief. If only those beliefs in which we have credence one are justified directly, then the reach of direct justification is very limited.

A second reason to hesitate before restricting direct justification to only the clearest experiences is that doing so fails to capture the gradual shift in phenomenology taking place in these cases. On this view, the very slight shift in degree of justification between $E_{t 1}$ and $E_{t 2}$ corresponds to a shift in the operationally relevant contents, from tree contents, to appearance properties and background information. And yet the phenomenal differences between $E_{t 1}$ and $E_{t 2}$ are so slight that it seems implausible that the justification $E_{t 1}$ offers for belief is of a different form, and in virtue of a different set of contents than the justification $E_{t 1}$ can offer. Given how small the phenomenal shift is between $E_{t 1}$ and $E_{t 2}$, if $E_{t 1}$ includes tree content, so should $E_{t 2}$. Of course we cannot reiterate that step indefinitely, but at this stage we are concerned with the smallest shift in phenomenal character that has an impact on the degree of justification. And that shift seems so small that if an experience on one side of it can directly justify a tree belief, so can an experience on the other. But if only the very clearest of experiences provide direct justification then there is a step-change in the nature of the justification provided by $E_{t 1}$ and $E_{t 2}$. That is because, on this view, the first time we have a change in degree of justification, we have a shift from an experience with tree content, to an experience on the basis of which Elmer can infer he's looking at a tree.

\section{iii. Low-level contents}

Another possible response to these cases is to observe that the tension they generate relies on the contested claim that visual experiences include highlevel contents, contents like trees, and cats. In giving up high-level contents, we give up the claim that the operationally relevant contents remain stable across epistemically relevant shifts in character. If low-level contents shift more closely in line with character, and if low-level contents are responsible for the direct justification of belief, then that direct justification too will respond to changes in the character of experience. On this view, the density of relevant low-level contents determines the strength of the inference from low-level contents to high-level contents, and hence the degree of justification the subject enjoys for beliefs about those higher-level contents. If I have only sparse low-level content consistent with a tree, then my inference to a tree is less well supported than when there is a higher concentration of tree-consistent contents. Could we avoid cases like those of Rey and Elmer by allowing that direct justification is properly restricted to beliefs about more basic contents, such as color, size or location properties? 
Accepting that visual experience represents only a more limited range of properties will limit the reach of these sorts of problem cases, but only so far as it sets corresponding limits on the range of external world propositions our experience can offer direct justification for. If visual experience represents a more limited set of experiences, then the range of possible cases of the kind described above is similarly restricted. The more interesting question is whether restricting visual contents in this way lets us avoid these sorts of cases altogether.

Merely retreating from high-level contents will not be enough to avoid these cases altogether. Shape, depth, color and location properties are generally treated as low-level properties, which are uncontroversially represented in perceptual experience. ${ }^{23}$ But even if we limit ourselves to those properties we can generate cases in which an individual intuitively has some limited degree of direct justification for belief about those properties.

Take Rey's experience. Without assuming high-level contents, or even that her experience represents the object in question as a box, we can still ask at what point her experience represents the shape or location of the box. At $E t_{n}$, when she has fully turned towards the object, she plausibly has direct justification for the beliefs that the object has a particular shape and location. At $E t_{n-1}$ her visual experience of the object is just marginally less clear. If the degree of justification she enjoys for belief is to track her developing phenomenology, then she should enjoy just slightly less justification for that same beliefs about the object's shape. But what explains that restriction? If the experience already represents the object's shape at this point, then it can directly justify her belief. And it is plausible that the experience does represent its shape. After all, it doesn't seem that Rey's experience $E t_{n-1}$ fails to represent shape, nor that it represents it as having a different, inaccurate shape. So that feature of the experiential content remains the same between $E t_{n}$ and $E t_{n-1}$, and it is that content which is operationally relevant in this case. But then that content fails to reflect the epistemically relevant change in the phenomenal character of the experience between these times.

I leave it open whether there may be some visual contents so basic that it seems inconceivable that our experience could justify anything less than full belief in them. Perhaps certain color properties could have that form, for instance. Allowing that possibility should not stop us recognizing that these problem cases can arise for other low-level properties.

\footnotetext{
${ }^{23}$ Prinz, for instance writes that "Just about everyone would agree that normally developing people can see certain spatial properties that are present in the distribution of light. These properties include color, shape, motion, and illumination" (2013:828).
} 
iv. Other changes in perceptual contents

A related response to these cases explains the shift in degree of justification on the basis of other changes in the contents of the experience, beyond the presence or absence of tree content. It's natural to offer the accumulating fog a role in determining the epistemic profile of the experience, or changes in the luminance and sharpness in the appearance of the tree that that leads to.

The tension described above does not demand that these changes are not epistemically significant. It only relies on the continuing epistemic significance of tree content, even as fog accrues. The phenomenal changes induced by the gathering fog are compatible with the experience continuing to have tree content, and with that content continuing to play a role in determining the epistemic capacities of the experience. The point is that trees can look a lot of different ways, whilst still looking like trees. They can be seen through rain and fog, at a distance or from an angle. Tree content is robust enough to withstand these kinds of variations. That tree content allows the experience to directly justify belief. We need an account then of how the epistemic impact of that tree content is non-inferentially impacted by the gathering fog.

\section{v. Indeterminate visual states}

By contrast with the options discussed above, what we want is to allow that some set of Elmer's experiences can provide direct justification for the belief that there is a tree outside, but that the experiences in that set need not all provide the same degree of justification for that belief. That variation in degree of justification reflects differences in the phenomenology of the two experiences. Put in terms of credences, the cases above elicit the response that a credence of less than one may be directly justified by a visual experience. The challenge is to explain what fixes the credence the experience is capable of directly justifying, and why some visual states justify higher credences in their contents than other visual states.

The problem we are confronted with is as follows: degree of justification can diminish gradually. That gradual change in perceptual justification reflects the gradual change in the phenomenal character of Elmer's experience. But so long as we treat tree content or location-content as all-or-nothing, we cannot capture that gradual shift, because direct justification via the endorsement of that content is all-or-nothing. So at some point there is a sudden shift in the kind of justification Elmer or Rey enjoys, from direct to inferential. The abruptness of that shift fails to reflect the changing nuance of their phenomenal experience. A satisfactory 
perceptual epistemology would allow the gradual change in phenomenal character of the experience to be mirrored by gradual changes in its epistemic capacities. But how can those epistemic capacities undergo a gradual transformation if they are rooted in the endorsement of binary perceptual states?

We are faced with a mismatch between epistemic capacities that supervene on all-or-nothing contents of visual states, and the gradually changing phenomenal character of visual experiences over time. In this way, the tension identified above stems from the assumption that a visual state either has a particular content or it lacks it. One way of resolving the tension identified above whilst preserving $D V J(E x W)$ is to give up on that assumption.

We could do this by simply describing the relevant contents as simply indeterminate. When Elmer sees a tree in fog, his experience has a content that is neither determinately a tree, nor determinately not-a-tree. In virtue of this his tree belief is less justified than when his visual state includes determinate tree content.

Simply describing the contents of his experience as indeterminate does not on its own give us the epistemic resources we need. An indeterminate experience plausibly offers weaker justification for belief than a determinate experience. We don't just want to recognize two levels of justification, however, but a host of incremental distinctions. In Elmer's case, we need to account for a smooth positive correlation between the dissipating fog and the degree of justification. Degrees of justification seem to require degrees of determinacy.

What does it mean for visual states to come with degrees of determinacy? One natural way of understanding this is to think of visual states as probabilistic. They don't simply represent an object as a tree, but as more or less likely to be a tree. As I argue below, this approach loosens up the puzzles presented above, and lets us offer a simple account of immediate justification of degreed belief states, or of different degrees of justification for outright belief. This allows us to respect the desideratum that our visual justification reflect the nuance of our visual phenomenology, whilst also allowing that we have different degrees of justification for different visual beliefs, or that different experiences justify different degrees of belief in their contents. We can do this in a way that protects the claim that visual beliefs may be directly, and even immediately justified in some instances.

\section{Visual confidences}

Visual states could be probabilistic either in virtue of having probabilistic contents or in virtue of containing a probabilistic relation to their contents. 
In what follows, I take the latter avenue, allowing that visual states include something like the perceptual analogue of credence for belief. ${ }^{24} \mathrm{~A}$ credence is an attitude a subject takes towards a particular content. In that sense it is a relation to contents, not part of the contents. Visual states too could include a confidence relation to their contents, in the manner of a probability operator. On this view, a visual state is made up of visual contents together with a set of confidence relations to that content. This allows that more or less determinate experiences can include the same contents as one another. What varies is the confidence relation they bear to that contents. I will call these degreed relations "visual confidences". The higher the degree of confidence, the higher the determinacy of the visual state.

On this view our visual experience does not just represent objects and properties, it also assigns degrees of confidence to those representations. Imagine walking along a beach towards an object in the distance, as it becomes gradually apparent to you that it is a turtle. As you advance along the beach your visual experience changes. Part of what changes is the confidence associated with the turtle contents. That confidence increases as you approach the turtle. That confidence relation is part of your visual experience.

A single visual experience includes states with a range of confidence levels. A tree in the foreground will be represented with a higher degree of confidence than one in the distance, or at the periphery of your visual field. It is not just that object-identification is subject to differing degrees of confidence. A visual experience can present the particular colors or shapes or locations of objects with differing degrees of confidence.

Differences in confidences are phenomenally detectable: a turtle represented with a high degree of confidence looks different to a turtle represented with a low degree of confidence. Recounting your experience on the beach, you might say "as I drew closer I could see it was probably a turtle washed up on the beach." Visual confidences allow that you really did see that it was probably a turtle. This talk reflects the fact that visual contents can come with more or less confidence assigned to them. In this way, visual confidences provide a powerful tool to describe some of the otherwise hard-to-capture distinctions in the contents of our visual experiences.

${ }^{24}$ In this respect my account aligns more closely with that of Morrison, (who talks of experiences assigning confidences to particular contents (forthcoming)) than with Moss (who talks in terms of visual experiences having probabilistic contents (ms).), though Morrison also considers the possibility of including degrees of confidence in the propositional content (forthcoming: 31f.). 
In this way, confidences can allow that there are changes in the phenomenal character of an experience which are not reflected in a change in content per se. Some shifts in character can instead track changes in the confidence associated with those contents. This introduces an important degree of freedom.

How do confidences apply to the cases of Elmer and Rey described above? Take Elmer's visual experience of a tree. There is some content common to his experience of a tree in moderate fog and his experience of a tree in minimal fog. They both represent a tree. But something varies across those experiences, in addition to the presence of fog. The gathering fog has an impact on how the tree itself is presented. As the fog gathers, the tree is represented with less confidence.

Consider the experience of Rey as she turns her head. What changes as she does so? The contents of her experience develop as what falls within her visual field changes, and the box moves from the periphery to the center of her visual field. At some point her experience unambiguously represents a grey object. When does that content appear? If we suppose that visual contents is all or nothing, its emergence represents an abrupt change in the contents of her experience. Allowing that visual states include a confidence relation lets us tell a different story. At some point her visual state includes a grey object among its contents, but that content is initially associated with a very low confidence. Part of what changes as she turns her head is the confidence with which the object is represented. The contents itself remains stable, but that changing confidence tracks the phenomenology of the experience as it develops over time.

Why have confidences been overlooked in work on perceptual content to date, even as work on belief has embraced them? One source of resistance may be the sense that I limit my confidence in response to information. Hence it is natural to think of belief in terms of varying degrees of confidence, because belief is a response to information. Visual information on the other hand has traditionally been seen as information, the information in response to which one might form a more or less confident belief. 25

One way of assuaging this worry is to notice that visual experience is itself a response to information, even as it serves us in turn as a source of information about the external world. Visual experience is a response to uncertain retinal information. Retinal data fails to offer a determinate representation of the world. That feature of visual experience is foregrounded in probabilistic models of perception which have gained

${ }^{25}$ I am indebted to Susanna Siegel for discussion of this point. 
ground recently in both philosophy and vision science. ${ }^{26}$ These models come in a range of flavors, but for our current purposes what matters is their agreement that visual experience represents a best guess on the part of the visual system, a kind of prediction of our external environment, informed and corrected by sparse retinal input which is incapable on its own of delivering a determinate representation of the distal stimuli that generate the experience in question. According to these models, our visual experience is merely the most likely interpretation of incoming retinal data in light of prior probability distributions. In effect, our visual experience represents some portion of the probability distribution. ${ }^{27}$ The claim made by these models that visual experience is merely probabilistic fits naturally with the claim that the contents of our visual experience are not limited to brute objects and properties but include associated degrees of confidence, confidences which reflect the probability that this particular representation gets it right.

We should not be too quick in making the leap from probabilistic models of the processes responsible for visual experience to the claim that the content of those experiences is associated with confidence levels. We should not pretend, for instance, that visual confidences allow us to say that visual states simply directly reflect the probability distribution generated by the visual system. It is not as though we can read off the information contained in that distribution from our experiences, nor even the fact that it involves a probability distribution over competing hypotheses at all, (or vision science might have arrived rather more quickly at these sorts of probabilistic models). Given that visual confidences are in part motivated by the desire to capture more accurately the subtleties of our visual experience, we cannot then claim that they include content so wholly alien to our visual phenomenology as an entire probability distribution. The point, rather, is that the visual system already deals in probabilities and graded confidences. Given that it is commonplace to think that the belief system does too, it becomes increasingly strained to think of visual experience as a binary mediator between the two of them,

\footnotetext{
${ }^{26}$ For accounts of this kind see Andy Clarke (2013), Jakob Hohwy (2013), Vetter and Newen (2014), James Feldman (2014) or Friston (2012).

27 According to Bayesian models of perception, visual perception produces as its output a set of probabilities distributed over competing hypotheses about the arrangement of the external world. As James Feldman (2014) observes, Bayesian models themselves provide no particular mandate for which part of that information visual experience should itself represent. That selection depends on the "loss function" an agent adopts. The maximum a posterior value, that is the peak of the probability distribution, will be selected when the loss function is zeroone, when "all incorrect responses are equally penalized and correct responses are not penalized at all" (Feldman 2014:17).
} 
talking, in effect, a different sort of language, in particular when doing so fails in addition to capture the nature of that experience.

In what follows I am concerned to explore how visual confidences play a role in belief-formation and justification. I will argue that visual confidences could play a normative justificatory role, by moderating the degree of justification I have for a visual belief. Put in terms of credences, they moderate the credence I am entitled to have in my visual beliefs.

\section{Visual confidences and direct justification}

I have suggested that visual confidences let us capture subtleties in how objects appear to us, and to Elmer and Rey in the cases above. I turn now to offering a more detailed account of how visual confidences could contribute to a simple and convincing story of how visual experiences can directly justify belief states. What that account looks like depends on whether we take the beliefs in question to be full beliefs, or credences. Let's assume in the first instance that those beliefs are full beliefs. How is it that visual experience can offer different degrees of direct justification to binary visual beliefs?

Suppose that visual contents take the form of structured propositions, such that a visual experience of a bear consists of a propertyobject pair of the form $<x$, Bear $>$ that attributes the property of being a bear to an object that features in the visual experience. Plenty will disagree with this supposition, but it at least allows us to set out in more depth a sample account of how visual confidences justify belief, which can then be adapted to one's favored model of perceptual contents. How does a confidence level come into this picture? A confidence level attaches to the attribution of the property to the object, that is, to the pair we already have. When I see a bear right in front of me, my visual experience has the contents $<x$, Bear>, and I bear a relation of, say, .98 confidence to that content. When I see a bear in the distance, my visual experience might involve a relation of .55 confidence to the contents $<x$, Bear $>$.

Visual confidences offer powerful epistemic resources, because they allow that an experience can include a particular contents without that implying that one should have high credence in the corresponding perceptual belief. The puzzles of Elmer and Rey above start from the observation that perceptual beliefs can be justified to a range of degrees. I may form the same belief, "that's a bear!" whether I see a bear close up or in the distance. But those beliefs enjoy differing degrees of justification: when I see the animal in the distance then the corresponding belief enjoys a weaker level of justification than when I get a good look at it up close. Visual confidences are well placed to explain this variation. They let us tie that difference in the degree of justification enjoyed by different perceptual 
beliefs to variation in the perceptual states that ground them. The contents of my visual experiences entitle me to form certain beliefs on their basis. The confidence with which those contents are presented moderates the degree of justification the associated beliefs enjoy. The distant bear experience associates the contents $<x$, Bear $>$ with a confidence of .55 and the associated visual belief is justified to a weaker degree than the belief associated with the close-up bear experience, when the content $<x$, Bear $>$ is associated with a confidence of .98 . The confidence relation explains how justification can continue to be immediate, whilst also coming in different degrees. Your belief uptakes the content of the visual experience, severed from its confidence relation. The degree of justification is determined by the confidence level. There is no need for any intervening inference between experience and belief on this picture.

On this picture, visual states are like weather forecasts that predict rain with a certain degree of confidence. If the weather forecast tells you there's a 50\% chance of rain tomorrow, that offers only moderate justification for the belief that it will rain tomorrow. Visual confidences allow that visual perceptual states mediate the justification of beliefs based upon them in a similar way. The confidence with which contents are represented determines the justification I have for the belief in question, just as the confidence with which rain is forecast determines the justification I have for the belief that it will rain tomorrow.

A visual confidence is in effect an estimate of the likely accuracy of the associated contents. If the visual system is well-calibrated, then the visual contents it associates with a .9 confidence will be accurate $90 \%$ of the time, whereas the contents associated with a .2 confidence will be accurate only $20 \%$ of the time. Those confidences amount to a representation of the likely accuracy of the associated contents. In virtue of that the first belief is more strongly justified than the latter.

Visual confidences give us the resources to describe the changing epistemic position of Elmer and Rey. The different experiences available to Elmer include states that represent trees with varying degrees of confidence. If Elmer goes to the window soon, his experience will represent a tree with a high degree of confidence. That confidence gradually drops away and at some point his experience no longer includes Tree content. But that is not an abrupt cut off. Just as the confidence associated with the Tree content gradually declines, so does the degree of justification those states would confer on Elmer's tree beliefs. The experiences can still directly justify tree beliefs. The justification is still in virtue of the relationship between the contents of the experiential state and the contents of the belief state. But the lower confidence with which the content is presented limits the degree of justification. In this way, visual confidences allow direct 
justification to mirror subtle and gradual shifts in the phenomenal character of our experiences.

We can tell a similar story about Rey: as she turns towards the box, the confidence associated with the visual states that represent it increases. They need not do so at the same pace: perhaps the experience clearly represents the color sooner than it does the size of the object. The confidence associated with each state determines the degree of justification of that aspect of her visual belief. The justification relation is still grounded in the experiential contents, but the relation is sensitive to the confidence with which the visual contents are presented. Visual confidences let us give an account of direct justification by visual experience that respects the desideratum on perceptual justification described in section one: they let us tell a story about justification that is responsive to differences in the nuance of how objects appear to us.

Accounting for these nuances in phenomenal character in terms of visual confidences need not overlook the significance of other changes in content, e.g. the accumulation of fog, or the changes in luminance and sharpness in the appearance of the tree that that leads to. Those other changes in content are not independent from the shifting confidence with which the tree is presented. It's the gathering fog and the decreasing luminance, for instance, which are responsible for the diminishing confidence you have in the tree content. But those features aren't incompatible with your experience continuing to have tree content or color and size content, nor with that content continuing to offer a form of direct justification for belief.

Visual confidences also allow that full beliefs about probabilities can be directly justified by visual experience. Visual contents associated with a lower degree of confidence justify the corresponding probabilistic belief: the contents $<x$, bear $>$ associated with .65 confidence justifies the belief that the object is probably a bear. Associated with a .35 confidence it could justify the belief that the object is possibly a bear. Both experiences still offer some support for full belief that the object is a bear, but to a more limited degree. Note that on this view there is no single belief that a visual experience offers direct justification for. It can justify a range of beliefs to different degrees depending on the strength of those beliefs. A belief that an object is possibly a bear is strongly justified by a visual state associated with a lower confidence, whereas the belief that the object is a bear would receive a weak degree of justification from the same state.

\section{Degrees of belief}

Part of the epistemic motivation we described for visual confidences came from the difficulty of accounting for the interface between binary 
experiential states and degreed belief. Our discussion since then has focused on the question of how visual states that include confidences can justify full beliefs. What if belief comes in degrees? How do degreed visual states justify belief in that case?

Visual confidences offer a simple solution to the problem of how visual experiences interface with graded belief states. Visual states with confidences are themselves graded. They can therefore interface directly with graded belief states. There is no mystery around what credence a given experience licenses in its contents. It offers prima facie justification for the credence which corresponds to the confidence relations associated with the relevant content. There is no need for beliefs about the experience to mediate the justification the experience offers belief. Visual confidences fix the credence the believer is entitled to have in the corresponding belief.

Take the case of Elmer. If he goes to the window soon, he will be able to see the tree outside clearly. That experience entitles him to have a high credence in the belief that there's a tree outside. If he waits a little longer, his foggier experience will entitle him to have only a moderate credence in that belief. Why? Both experiences have tree content. But that content is presented with different degrees of confidence. When it is presented with a high degree of confidence, that entitles Elmer to have a correspondingly high credence in the corresponding belief. When it is presented with a moderate confidence, that entitles him to have the same middling degree of belief in its contents.

We can draw again on the analogy with a weather forecast. If the weather forecast tells you there's a 50\% chance of rain tomorrow, that licenses a .5 credence in the proposition it will rain tomorrow. The confidence with which contents are represented determines the credence with which I am entitled to hold the belief in question, similarly to the way in which the confidence with which rain is forecast determines the credence I am entitled to have that it will rain tomorrow. It is appropriate for Elmer to have a higher credence in the clear experience contents than the foggy experience contents: the higher confidence associated with the former represents a higher likelihood that they are accurate, (provided the system is reasonably well-calibrated). As a consequence, those different credences constitute an appropriate response to the evidence provided by those visual states.

Both high and low credences can be equally justified, in virtue of their correspondence with the relevant visual confidence. ${ }^{28}$ What if the

\footnotetext{
${ }^{28}$ By forming credences that are in line with well-calibrated visual confidences an individual will in effect be conforming to a version of David Lewis' (1980) Principal Principle, according to which a rational agent conforms their subjective credences to the objective chances.
} 
agent fails to conform their credence to the confidence of their experience? In that case we can evaluate the degree of justification that the credence they do form enjoys. The further it departs from the credence that is warranted by the experience, the weaker its justification.

I have claimed that visual experiences justify credences that correspond to the confidence associated with their content. But there are times when this claim seems obviously implausible. Consider cases of visual illusion, for instance. The apparent bend in the stick as it enters the water, or the lines of the Muller-Lyer illusion might be presented with a very high degree of confidence, even after I know that I am subject to a visual illusion. It seems obviously wrong to claim I continue to receive the same degree of justification for the belief that the stick is bent or the lines different lengths to one another.

In these cases, we can recognize that you continue to receive the same degree of prima facie justification from your visual experience for the belief that the stick is bent. But that does not mean that that belief is ultima facie justified. In these cases of visual illusion there is a defeater for the prima facie justification - your awareness that your visual experience is misrepresenting certain aspects of your environment.

The account presented here describes the justification visual states provide for belief in the absence of defeaters or other relevant background features. It describes only the simplest way in which visual states can justify belief states. Very often the epistemic impact of an experience depends not just on the visual states that comprise it, but on prior information that lets the agent update appropriately on the basis of that novel experience. That information may be cognitive, or it may consist of other visual experiences, and the way such experiences develop dynamic relations with one another over time.

\section{Comparative and disjunctive beliefs}

It is significant that once we grant that visual states include a range of contents associated with confidence levels, it is no longer the case that a visual state justifies a unique belief state in virtue of its contents. This has some important upshots for the resulting picture of direct visual justification.

This feature of the account has the result that a single visual experience may directly justify inconsistent propositions. Consider the cases offered in Silins (2011) of a visual experience of a card with dots on it, that is flashed before you. On the basis of your visual experience you cannot be sure if the card has ten or eleven dots on it. Nonetheless your experience gives you justification to raise your credence in both those propositions. As a consequence, "you gain immediate justification to 
increase your credence in multiple propositions which cannot all be true." Silins uses these cases to argue against the content constraint, noting that "[s]o long as the content of these experiences remains consistent, the propositions justified by the experience will outrun their content" [2011: 346].

Once we allow confidences into the picture we can explain these cases in the following way. Your visual experience of the card includes both ten-dot and eleven-dot content. Each of those contents is associated with a low degree of confidence. Supposing that those confidences are higher than your existing credences that the card has ten dots or that it has eleven dots, then your experience justifies you in raising your credences in both propositions in response to it. That increase in credence in conflicting propositions is an appropriate response to the content of the experience. As a result, once we allow that a visual state includes a range of contents associated with distinct degrees of confidence, these cases no longer provide a counter example to the content constraint. You may have direct justification to raise your credence in multiple inconsistent propositions on the basis of a single visual experience, when your experience includes a range of potentially inconsistent contents with different degrees of confidence.

Visual confidences also allow us to describe what happens when you see a card fleetingly with a slightly higher or lower number of dots. The character of your experience changes in some way as the number of dots changes. That change in character can be accounted for in two ways. On the one hand, it could be due to a shift in the propositions over which your experience distributes in confidences. When the card in fact has ten dots then perhaps your confidence is distributed over propositions that range between eight and twelve dots. When the card in fact has eleven dots then it is plausible that that range shifts slightly higher, to, say, between 9 and 13 dots. Alternatively, that change in character could be accounted for by a change in the confidence relation your visual experience bears to the same set of propositions. The phenomenology of an experience will reflect the shape and peak of the probability distribution as well as its boundaries.

Visual confidences also allow that visual experiences can offer direct justification for comparative or disjunctive beliefs, for instance that an object is either blue or purple, or is more likely blue than purple. Both Silins (2011:350) and Pautz (2011:397) doubt that visual experiences could have comparative content of a sort that could directly justify such beliefs. But suppose you have a visual state which represents an object as blue with .6 confidence and as purple with .3 confidence. If one simply endorsed that experience in belief, one would have direct justification for a higher credence that the object is blue than that it is purple. This is 
equivalent to the experiential state offering direct justification for the comparative belief that the object is more likely blue than purple. Suppose you have a visual experience with confidence distributed evenly over two competing possibility, for instance, that an object is a dog or that it is a goat. Your experience in effect offers direct justification for the disjunctive belief that the object is either a dog or a goat. Visual confidences have the result that a single visual experience justifies complex beliefs directly, in virtue of its contents and the confidence relations we bear to them.

\section{Conclusion}

Visual confidences offer a range of powerful resources to help us make sense of the epistemic relationship between visual states and belief states. They let us offer an account of how the former come to inform and justify the latter. This account can allow both that direct justification can come in degrees, and that credal states can be immediately justified by visual experience. Visual confidences thereby allow us to capture the way in which variations in our visual phenomenology result in subtle variations in the kind and degree of justification we have for belief.

\section{Works cited}

Alston, William (1989). Epistemic Justification. Cornell University Press.

Audi, Robert (1993). The Structure of Justification. Cambridge University Press.

Bayne, Tim (2009). Perception and the reach of phenomenal content. Philosophical Quarterly 59 (236):385-404.

Block, Ned (2010). Attention and mental paint. Philosophical Issues 20 (1):2363.

Brewer, Bill (2006). Perception and content. European Journal of Philosophy 14 (2):165-181.

Brogaard, Berit (2013). Do we perceive natural kind properties? Philosophical Studies 162 (1):35 - 42.

Chalmers, David J. (2006). Perception and the fall from Eden. In Tamar S. Gendler \& John Hawthorne (eds.), Perceptual Experience. Oxford University Press 49-125.

Chalmers, David J. (2010). The Character of Consciousness. Oxford University Press

Christensen, David (2004). Putting Logic in its Place: Formal Constraints on Rational Belief. Oxford University Press.

Clark, Andy (2013). Whatever next? Predictive brains, situated agents, and the future of cognitive science. Behavioral and Brain Sciences 36 (3):181-204. 
Dretske, Fred (1995). Naturalizing the Mind. MIT Press.

Feldman, J. 2014. Bayesian Models of Perceptual Organization in J. Wagemans (ed.) Oxford Handbook of Perceptual Organization. Oxford, OUP.

Feldman, Richard. (2003). Epistemology. Pearson.

Frankish, Keith (2009). Partial Belief and Flat-out Belief. In Franz Huber \& Christoph Schmidt-Petri (eds.), Degrees of Belief. Springer 75--93.

Friston, K. 2012. A Free Energy Principle for Biological Systems. Entropy 14 2100-2121.

Goldman, Alvin. (1979). “What is Justified Belief?” In G. Pappas (ed.) Justification and Knowledge Dordrecht: Reidel

Goldman, Alvin I. (2008). Immediate justification and process reliabilism. In Quentin Smith (ed.), Epistemology: New Essays. Oxford University Press 63--82.

Hawley, Katherine, and McPherson, Fiona, (eds.) 2011. The Admissable Content of Perception. Wiley-Blackwell.

Hill, Christopher S. (2014). Meaning, Mind, and Knowledge. OUP Oxford.

Hohwy, Jacob. 2013. The Predictive Mind. Oxford, OUP

Huemer, Michael (2001). Skepticism and the Veil of Perception. Lanham: Rowman \& Littlefield.

Jeffrey, Richard. 1965. The Logic of Decision. University of Chicago Press.

Joyce, James M. (2005). How Probabilities Reflect Evidence. Philosophical Perspectives 19.1 153-178

Lewis, David (1980). A subjectivist's guide to objective chance. In Richard C. Jeffrey (ed.), Studies in Inductive Logic and Probability. University of California Press 83--132.

McGrath, Matthew. Forthcoming. Looks and perceptual justification. Philosophy and Phenomenological Research.

Morrison, John. Forthcoming. Perceptual Confidence. Analytic Philosophy

Moss, Sarah. ms. Probabilistic Knowledge. Draft of 02/05/16

Pace, Michael (2010). Foundationally justified perceptual beliefs and the problem of the speckled hen. Pacific Philosophical Quarterly 91 (3):401-441.

Pautz, Adam (2011). Can disjunctivists explain our access to the sensible world? Philosophical Issues 21 (1):384-433.

Pautz, Adam (2015). What is my evidence that here is a cup? Philosophical Studies:1-13.

Prinz, Jesse (2013). Siegel's get rich quick scheme. Philosophical Studies 163 (3):827-835.

Pryor, James (2000). The skeptic and the dogmatist. Nô̂s 34 (4):517-549.

Pryor, James (2005). There is immediate justification. In Matthias Steup \& Ernest Sosa (eds.), Contemporary Debates in Epistemology. Blackwell 
181--202.

Rescorla, Michael. (2014). Can perception halt the regress of justification? In J. Turri and P.D. Klein (eds.) Ad Infinitum: New Essays on Epistemic Infinitism. Oxford University Press.

Schellenberg, Susanna (2014). The epistemic force of perceptual experience. Philosophical Studies 170 (1):87-100.

Schellenberg, Susanna (forthcoming). Phenomenal Evidence and Factive Evidence Defended. Philosophical Studies:1-18.

Siegel, Susanna (2006). Which properties are represented in perception? In Tamar S. Gendler \& John Hawthorne (eds.), Perceptual Experience. Oxford University Press 481--503.

Siegel, Susanna (2006b). Subject and object in the contents of visual experience. Philosophical Review 115 (3):355--88.

Siegel, Susanna (2013). Can Selection Effects on Experience Influence its Rational Role? In Tamar Gendler (ed.), Oxford Studies in Epistemology volume 4. Oxford 240.

Siegel, Susanna \& Silins, Nicholas (2015). The Epistemology of Perception. In Mohan Matthen (ed.), Oxford Handbook of Philosophy of Perception. Oxford

Silins, Nicholas (2011). Seeing Through the 'Veil of Perception'. Mind 120 (478):329-367.

Skyrms, B. (1977). Resilience, propensities and causal necessity. Journal of Philosophy 74.11 704-713

Travis, Charles. S. (2004). The silence of the senses. Mind 113 (449):57-94.

Tye, Michael (1995). Ten Problems of Consciousness: A Representational Theory of the Phenomenal Mind. MIT Press.

Vetter, Petra \& Newen, Albert (2014). Varieties of cognitive penetration in visual perception. Consciousness and Cognition 27:62-75.

Williamson, Timothy (2000). Knowledge and its Limits. Oxford University Press.

Wright, Crispin (2007). The perils of dogmatism. In Nuccetelli \& Seay (eds.), Themes from G. E. Moore: New Essays in Epistemology. Oxford University Press 